\title{
PURPLE OCEAN STRATEGY: HOW TO SUPPORT SMES' RECOVERY
}

\author{
Giorgio Gandellini \\ Daniela Venanzi \\ University of Rome III, Italy
}

\begin{abstract}
This mainly conceptual paper suggests a new approach, aimed at supporting SMEs' strategic realignment in the recovery phase after the economic crisis of 2008-2009. The analysis of the impact of the recent crisis on the performance of the Italian medium-size firms [MSEs], allows the identification of the levers on which their recovery can be based. Italian MSEs represent an interesting laboratory for experimentation for many reasons. In particular, their experiences in reacting to the crisis show the critical importance of strategic moves enhancing the competitive profile in terms of value of the firm's investments, and can be easily applied to their SMEs counterparts, provided that specific and rigorous methodological guidelines are identified for their practical application.
\end{abstract}

The model suggested in this article, starting from the Blue Ocean Strategy's approach, identifies the weaknesses of its original formulation, and proposes its integration and development, aimed at providing SMEs with a more articulated and systematic framework (i.e. the "strategic and economic control dashboard" or, in short, Strateco Dashboard $\left.{ }^{\circledR}\right)$, that equips managers with the qualitative and quantitative tools for "piloting" the key competitive success factors [KCSFs], in terms of both managing the components of value, and measuring its economic and financial impact.

In particular, the methodology suggested in the article introduces three operational and quantifiable constructs: (a) the "investment curve", which specifies the actual amount of resources invested in the tools that have an impact on the KCSFs, (b) the actual "value" generated by these investments, based on the relative importance attributed by the market to these KCSFs, and (c) the relative "value/price ratio", that has an impact on the firm's market position, based on assumptions about the demand elasticity and the expected competitors' behavior, if any.

Furthermore, our methodology complements the assessment of the market value of strategy with a construct that estimates its impact on financial performance, in terms of value creation. With appropriate metrics, we develop the logical approach for translating the investment curve and the valuel price ratio into the main value drivers, and therefore measure the value created by the strategy. Specifically, our attention is focused on the "surplus" value: it is not how much income a firm makes that marks its success, but how much it makes in excess of its cost of capital.

Keywords: strategies for facing crises; Blue Ocean Strategy (BOS); small and medium-sized firms (SMEs); decision support systems; value creation of strategy; economic crisis; financial feasibility of strategy; strategic management simulation.

\section{INTRODUCTION: THE 2008-2009 CRISIS, AND THE ITALIAN MANUFACTURING MSEs}

The Italian manufacturing MSEs, defined according to the definition of Mediobanca (the leading Italian investment bank) and Unioncamere (the national Association of the Italian Chambers of Commerce), which analyze them every year on a census basis, are companies which belong to the class of 50-499 employees (the median size being about 107 employees), have annual sales of $€ 13-290$ million and autonomous ownership (i.e. excluding companies controlled by or affiliated to large corporations, and non-national companies). Industrial groups are included as long as they meet the same size requirements. These companies represent an interesting laboratory for analyzing the impact of the recent economic crisis and highlighting the key strategic moves for a recovery.

A recent survey of MSEs in Europe (Confindustria-R\&S-Unioncamere, 2010), including 4,322 manufacturing MSEs in Italy, 1,472 in Germany and 616 in Spain, shows the following findings: 
a) the crucial role of the European MSEs and their relative importance in the aggregate national economies. In Italy, for example, the MSEs' percentage of manufacturing employment is $31.2 \%$, while the share of value added in manufacturing is at least $18.3 \%$ for corporations, reaching $30 \%$ if we include linked activities induced by MSEs

b) the economic trends are better for MSEs than for large firms in term of sales, exports, value added and employment during the analyzed period (1998-2007)

c) MSEs are concentrated in the most industrialized regions of the three countries: $80 \%$ in the Italian Northern, Eastern and Central regions, 79\% of their class' value added in the German Western and Southern regions, and $67 \%$ of the related manufacturing employment in the Spanish Catalonia, Basque, Valencia and Madrid areas.

Summing up, we can affirm that the analysis of MSEs' behavior and performance allows us to interpret the economic structure of a country better than with the traditional dualistic approach (small vs. large business), and to evaluate more effectively the challenges and opportunities to be faced in restructuring and increasing the competitiveness of the national economies. This is even more true for the Italian manufacturing MSEs, since they represent the core distinctive competences of the Italian economy for at least two reasons: (a) they operate in the Italian style industries, such as mechanical engineering, personal and household goods, and food; (b) about $25 \%$ of them are district firms, i.e. located in the so-called (by the Italian Institute of Statistics) industrial districts (IDs), but this proportion grows to $66 \%$ if we adopt a wider definition of district, based on proximity and business specialization criteria: lean production, chain organization, and network relationships based on franchising and similar distribution agreements.

Furthermore, the focus on Italian MSEs is even more justified if our concern is on investigating strategies of recovery from the current economic crisis. Many considerations support this choice. Firstly, as demonstrated by the IMF's last estimates, the Italian GNP index (2001 base) shows an upturn that is weaker than that of other industrialized countries. Secondly, MSEs contribute significantly and virtuously to the Italian economy: the universe of 4,483 manufacturing MSEs (less than $0.6 \%$ of the Italian companies) accounts for $18 \%$ of the national manufacturing industry's value added in $2007(30 \%$ if we consider the linked industries) and more than $17 \%$ of exports. These companies outperformed their larger counterparts, have a stronger competitive advantage and a negligible annual default rate $(0.4 \%$ vs. a national average of $1.4 \%)$ (Coltorti, 2009). Thirdly, the business model of the Italian MSEs is founded on the concept of "business as a life plan", characterized by the following features: family ownership and founder's entrepreneurship, focused strategy and leadership, brand identity, and price premium (Coltorti, 2008). These peculiarities shape their strategic management approach as a vital process for efficiently allocating the scarce financial resources and continuously renewing and feeding the competitive advantage. Fourthly, these companies were severely damaged by the 20082009 economic crisis, because of their export orientation (on average, $40 \%$ of their revenues), losing in that period $16 \%$ of value added, $30 \%$ of exports, and $15 \%$ of net operating margin (Coltorti, 2010). Finally, they are guiding the recovery from the crisis, by adopting a diversified process of strategic repositioning.

The in-depth analysis of the evidence from the Unioncamere survey (Gagliardi, 2010), conducted on a representative sample of the Italian manufacturing MSEs' universe, shows the following trends and recovery strategies:

a) about $80 \%$ of MSEs invested in 2009 and/or planned to invest during 2010

b) MSEs planned to buy more and make less and better: new investments were not aimed at operations, but at upstream and/or downstream activities, characterized by larger added value, intangible content and a stronger impact on durable competitive advantage. About $60 \%$ of the interviewed firms planned to invest in strategic assets like product quality, design and product innovation; $16.5 \%$ of them were stipulating network agreements for distribution and technology transfer; $66 \%$ planned to develop new products while $26 \%$ to outsource the most standardized phases of the value chain

c) exports guide the recovery of MSEs: $31 \%$ of the interviewed firms expected for 2010 an increase of international sales vs. $24 \%$ that expected an increase of domestic sales; on average, during 2009-2010 one third of MSEs had stable or increasing exports vs. one fourth that had stable or increasing sales 
d) the fall of the international demand pushed MSEs to re-orient their presence in the international markets; in particular, the percentage of MSEs which sell both in the EU and extra EU markets increased from $46 \%$ to $70 \%$, showing an important market diversification trend

e) the decrease of the operating margins will limit the percentage of self-financed new investments (53\%), while $40 \%$ of the investing MSEs will recur to debt: $27 \%$ of them experienced credit rationing, in terms of a decrease in credit limits $(28 \%)$, an increase in required guarantees $(26 \%)$, or a rejection (14\%).

We can conclude that the empirical evidence emerging from the analysis of the Italian manufacturing MSEs' recovery strategies, and the consideration of their distinctive characteristics, show the critical importance of the following key strategic moves:

- Emphasis on quality, design, and new products development, vs. penetration pricing strategies and reduction of the price-premium factor.

- Investigation and identification of new markets and products with more attractive value/price ratios.

- Focus on strategy's value creation: a more efficient use of the invested capital, due to its scarcity and cost (and therefore more attention to the capital charge and to the spread between return and cost of capital), and better orientation to the medium-long term, in order to (re)create a durable competitive advantage.

Based on these conclusions, our suggestions are therefore focused on the identification of a model that, inspired to the MSEs' strategies and the above mentioned guidelines, could be easily adopted by SMEs, that feel even more dramatically the need for a better and more selective allocation of their scarce resources. As a matter of fact, statistics (and common sense!) show that SMEs are nothing less than potential or former MSEs: in the 1998-2007 period the MSEs universe experienced 3,758 new entries (former SMEs) and 1,843 cancellations ("returning" SMEs).

In view of our objective, we found particularly helpful the following approaches:

- On the one side, the so-called Blue Ocean Strategy [BOS] model (Kim \& Mauborgne, 2005), which provides some useful guidelines for the identification and exploitation of relatively "uncontested" market spaces, thanks to an appropriate combination of innovative products and services, attractive value/price ratios, and selective use of the available resources.

- On the other side, an integration and adjustment of this approach with a more systematic model ( Gandellini \& Venanzi, 2011) that eliminates some of its evident methodological weaknesses, and provides an integrated and consistent framework for addressing the focused and selective strategic planning process badly needed by SMEs, not necessarily in Blue Oceans (without competitors), but also in "Purple" Oceans, in which the blue waters are frequently tarnished by the blood of the competitive war.

After a short summary of the BOS approach and a discussion of its inherent weaknesses, we will suggest some guidelines for a more systematic approach, remind the importance of appropriate quantification, propose an articulated and integrated conceptual and operational framework for the identification, development, and control of any possible strategy, in any industry and competitive context, and finally summarize the results of a "laboratory" experimentation of this approach, together with an indication of its potential improvements.

You will forgive us if, in order to explicitly differentiate our suggestions from those proposed by the managerial literature (BOS included), we will also indulge in the practice of adopting some new labels and acronyms.

\section{THE BOS MODEL}

The famous book by Chan Kim and Renée Mauborgne (2005), Blue Ocean Strategy, had an incredible worldwide success: doing business in "blue oceans", and avoiding the blood flowing from the competitive war (the "red oceans"), is apparently the new "must", or at least an objective to pursue.

It certainly was a "technically" excellent marketing endeavour, based on the expedient of an original title, an intriguing subtitle, new labels for several old concepts and similar views expressed by other authors (Beckwith, 1997; Porter, 1996; Howard \& Sheth, 1969; Levitt, 1975; Abell, 1980), an intelli- 
gent and well targeted communication campaign, and the prestige of the sponsoring business schools (Harvard and INSEAD). However, any reader of the book cannot avoid realizing that the statement "... make the competition irrelevant" is, in the best case, a little bold, even in specific market contexts mentioned by the authors in support of their thesis, and even more so in periods of economic crisis.

The major contribution of the book is that of having explicitly attempted a comprehensive systematization of the strategic process that allows the identification and exploitation of new industry sectors and market spaces, in which, at least initially, a competitive system does not exist by definition. Or, alternatively (and this is obviously the most frequent and relatively "within reach" case), the identification of new moves that displace the competitors at least for some time (this was precisely the case of Michael Dell, mentioned in the book as an example of BOS: direct sales in an industry traditionally based on retailing via computer shops).

To this end, the authors suggest a series of methodologies, more or less practical and effective. In particular, we found useful the analytical method for identifying the "obstacles to the purchase or use of the products or services", based on the phases of the "clients' experience cycle" (besides, largely known since the last century's seventies under the label of "buyer behavior": from the purchase to the use and disposal of the products), and the so-called "utility levers" (productivity, simplicity, convenience, risk, fun and image, environmental friendliness).

In short, the suggested methodologies have the purpose of allowing the identification and implementation of a "strategic move" based on the so-called "value innovation", consisting in delivering a high value at affordable prices that, however, allow the "blue ocean firm" (or, we should add, any firm, including SMEs) the creation of profits, thanks to both the size of the "new" demand (or a better exploitation of the existing demand), and the high selectivity and focus of its investments only on specific areas (choice criteria adopted by the market and/or resources that satisfy these criteria), definitely neglecting other areas.

\section{The "Value Curve" Approach}

Beyond the fact that the BOS approach dismisses the critical importance of quantifying estimates and projections in favor of more qualitative and less systematic methods, the major logical and methodological (and, consequently, practical) weakness we found in the authors' suggestions, on which most of their approach is based, is that of grounding, to a large extent, the identification of firms' "strategic moves" on the analysis of the so-called "strategy canvasses" and the related "value curves", which have the purpose of graphically describing the competitors' strategies in any existing industry, as well as the strategy that instead could be adopted by a company which wants to distinguish itself and innovate, thanks to the selective approach described above.

As an example, in Exhibit 1, adapted from the original Blue Ocean Strategy book (we added the "investment levels" label to the vertical axis, since it is explicit in the text, but not included in the original graph, but, like the authors, we did not define the meaning of the horizontal axis!), the authors compare the strategies of two major "strategic groups" in the U.S. wine industry (premium and budget wines) to that of the innovative (or, at least, presented as such) Australian Casella's wine [yellow tail], which focuses on new components of the offer (the three items at the bottom right of the figure), practically canceling the investments in other resources (the three on the left, price excluded): " $a$ fun and simple wine to be enjoyed every day". A similar approach is adopted for describing many other industry sectors, private and public, profit and non-profit.

\section{EXHIBIT 1}

Unfortunately, we do not really see how this approach could work in practice, since, in our view, it is fundamentally and seriously flawed from several standpoints. After having identified and discussed the major shortcomings of this method, we will propose ways of making it feasible, and, therefore, practically useful.

\section{The Major Problems with the "Value Curve" Approach}

In addition to other inaccuracies described in our working paper (Gandellini \& Venanzi, 2011), we found, at least, four major problems:

1. First, this approach does not distinguish between choice criteria adopted by the market and resources or tools in which the company actually invests, but these two aspects are treated on a par 
and mixed together: "ease of selection" and "fun/adventure" could be considered as choice criteria (manageable with investments in product range and distribution on the one side, and with appropriate communication approaches on the other), while "a-t-l marketing" is actually a tool in which the suppliers can invest (it is certainly not a choice criterion adopted by the market, but rather an element that could facilitate the choice, based on any possible criterion), and "wine complexity" or "aging quality" could be both criteria and investments, depending on the perspective from which they are considered (buyers' or suppliers'). An immediate consequence of this type of confusion is the impossibility of assessing the relative importance of the choice criteria on the one hand, and that of the resources in which to invest on the other.

2. The adopted scale (low or high investments, or, in any case, low or high "degree of focalization" or "emphasis") does not allow meaningful comparisons, even from a relative standpoint, for each component of the offer: does stating that [yellow tail] invests (or focuses) a lot on "easy drinking", "ease of selection" and "fun/adventure", totally disregarding the "technicalities in communication", make sense, while the current competitors are at rock-bottom on the three first items? Evidently, it is most of all (if not only) a matter of different positioning from a communication standpoint, irrespective of the amount of resources invested in communication. Besides, how could we imply that any new or light wine (most of the "budget" wines) is not "easy to drink"? Not to mention the fact that [yellow tail] does not seem so "easy" to drink ( $13.5^{\circ}$ of alcohol for the red variety), and its price is much closer to that of the premium wines. Furthermore, whatever the meaning of the vertical axis (investment levels or degree of focalization), the "price" scale should be reversed vs. that of the other components of the curve: a low price means a high level of investment or focalization/penetration, and vice versa for a high price, that allows much better margins.

3. But, most of all, it is totally impossible to hook up these value curves to the offers' economic profile (which is not taken into consideration in any part of the book, together with methods for estimating the market potentials): also because of the mixture and confusion of the concepts of "investment" and "choice criterion" (first problem), and the adoption of a purely qualitative scale (second problem), it is impossible to estimate in advance whether or not the value innovation will stand up from that viewpoint, i.e. if it will be able to generate wealth, thanks to an appropriate balance among value, prices, volumes, and costs.

4. Finally, and this is not a minor aspect, the authors intentionally exclude from the list of the possible components of the value curve (i.e. some items on its horizontal axis) the "brand image". The reason is clear: since they maintain "by definition" that in the blue oceans the competition does not exist, the "brand" is irrelevant. Unfortunately, anybody can see that this is a simplistic conclusion: the brand image is a fundamental element for identifying the suppliers, especially in "reddish" or "light-blue-but-not-so-much" oceans (Dell case), but also in oceans that are really blue at the beginning, and are subsequently entered by competitors: let us think of the mobile phones case, both at the dawn of the industry and, more recently, with the "smart phones" complemented and improved by Apple with significant innovations (touch screen, multitouch, accelerometer, ...). Apple succeeded, and still succeeds, also because of its brand name. As a matter of fact, we see how important are also the synergies among businesses (totally ignored by the authors) precisely thanks, to a large extent, to brand awareness and image: the creation, by Apple, of the "MP3" blue ocean with the launch of the iPod and the iTunes store (that, by the way, canceled from the face of the earth, in few months, the previous blue ocean created by Sony with the walkman), enormously facilitated the development of the new blue ocean, thanks to its iPhone. Same reasoning with the $i \mathrm{Pad}$, etc.

\section{A MORE SYSTEMATIC APPROACH}

In our view, the potential strategic opportunities faced by any company should be analyzed more rigorously and systematically, not just in the most frequent cases in which a competitive context already exists, but also when new markets are created (needs previously unmet by any industry) or new demand spaces in the context of existing sectors are identified.

1. Firstly, it is important to explicitly distinguish:

- $\quad$ on the one hand, the choice criteria that we expect will (or could) be adopted by the market, i.e. the components of the perceived or perceivable value, for example, and simplifying:

a. brand image 
b. product quality (in terms of functional performance)

c. product accessibility (in terms of availability and traceableness)

- on the other hand, the resources or tools in which the company should invest in order to satisfy these criteria; for example, and simplifying:

a. R\&D, raw materials, components, and manufacturing processes for managing quality

b. again, raw materials and components ("visible" by the market in many industries), type of distribution channels, advertising and promotion for managing brand image

c. distribution channels and sales force, credit terms, logistics, and, at least in part, again advertising and promotion for managing product accessibility.

2. Secondly, both for the choice criteria and the tools, it is important to estimate their relative importance: of the former for the market, of the latter for the components of value that they are supposed to manage (for the sake of simplicity, we assume that both the criteria and the tools are independent from each other: a consideration of their interrelationships would require a more sophisticated model which, however, would not add significantly to the conclusions).

3. Furthermore, for each type of resource or tool, and for the price, it is equally important to estimate, based on our own experience, intuition, and judgment, especially in the absence of an indepth knowledge of the industry (in case of radical innovations), a "reasonable" range of variability between minimum and maximum investment, expense, or price levels: i.e. a minimum level below which it would be useless to invest, since our efforts would be ineffective, and a maximum level beyond which we would obtain diminishing returns.

4. Finally, it will be important to develop equally reasonable estimates about the demand size, at a given value level, depending on extreme and intermediate price levels: i.e. assuming, although tentatively, possible "demand curves".

\section{The Need For Appropriate Quantification}

Overall, it will be necessary to "quantify" in some way the estimates, in order to be able to control the performance of the suggested strategies. As a matter of fact, contrary to the purely qualitative approach ("high" vs. "low") suggested in the Blue Ocean book, and its insistence on avoiding quantification ("focus on the big picture, not the numbers"), we are convinced that it is impossible to manage what cannot be measured, and totally share H.J. Harrington's opinion (1987): "If we do not measure we cannot control, if we do not control we cannot manage, if we do not manage we cannot improve".

Beyond the often provocative opinions expressed by some authors about the supposed inadequacy of numbers, everybody knows that, in order to correctly "pilot" a company (especially if it operates in multiple sectors), a strategic control system (in addition to the traditional economic and financial control) is indispensable, and cannot be based only on qualitative opinions (see, for example the "Balanced Score-Card" approach: Kaplan \& Norton, 1996). We are not looking for precision, but for relevance: in any case, decisions are made for the future, and precise data about the future do not exist by definition. Quantifying our estimates (imprecise by nature), assumptions, and hypotheses, is the only way we can make explicit our decision making process, communicate, discuss, and negotiate our conclusions, and control the discrepancies between projections and actual outcomes, in order to be able to adjust our strategies for the future.

\section{An Integrated "Strategic And Economic Control Dashboard"}

Based on the four guidelines described above, it will be relatively easy to design an articulated "strategic and economic control dashboard" ("Strateco Dashboard" ing basic elements (and not only of an approximate "value curve" that, as we have seen, is NOT "easy to understand and communicate for effective execution", as stated in the Blue Ocean book):

1. The "investment curve" ${ }^{\circledR}$ : numerical and graphical representation of "actual" alternative investment or expense levels (we use the term "investment" in a broad sense) in the above mentioned resources (see Exhibit 2). The investment indices for each resource or tool (e.g. raw materials, $\mathrm{R} \& \mathrm{D}$, and production systems), will serve the purpose of assessing the weighted average invest- 
ment in the various components of value perceived by the market (e.g. quality, in this case), based on the relative importance of the necessary resources or tools for managing the related components of value.

As we can see from the figure, alternative "investment" and pricing decisions (in practice, the strategies adopted in terms of resource allocation to the various tools) can be easily compared, thanks to a standardization on a scale 1-10 (based on the minimum and maximum levels previously estimated for each tool, and using, for the sake of simplicity, a linear function) of each individual decision.

\section{EXHIBIT 2}

We assigned to the minimum investment levels the index of 1 (meaning that even the minimum amount of invested resources could be somewhat effective, otherwise it would be wiser not to invest at all), to the maximum investment levels the index of 10, while the indices for the intermediate levels are proportional to the actual min-max range. In this case, a relatively low investment in price correctly indicates a relatively higher price level, and vice versa. By the way, the function that interpolates the extreme levels could be more correctly represented by a logistic (Sshaped) curve, but normally we do not have sufficiently reliable data for interpreting it, and, in any case, the basic trend would not change significantly.

Finally, we should say that, even though the min-max ranges of investments are based on estimates, the figures presented in this graphical framework are quite precise, since they correspond to "actual" investment decisions (see Appendix).

2. The estimated competitive profile in terms of value and the "value/investment compass ${ }^{\circledR}$ ". Based on an estimate of the relative importance of the various resources or tools for the individual components of value, presented in Exhibit 3 below (price excluded), the investment levels in each tool, and the relative importance of the components of value for the market, we can calculate a weighted average index of perceived competitive profile in terms of value (" $p c p V^{\text {, }}$ for short), always on a 1-10 scale. Considering these aspects, we can therefore design what we call the "value/investment compass", from which we can assess the degree of coherence between:

- the importance presumably assigned by the market to the components of value ( $\mathrm{X}$ axes in Exhibit 4 below): in this case we assume that service is most important, and that brand image and quality follow behind

\section{EXHIBIT 3 \\ EXHIBIT 4}

- $\quad \ldots$ and the related company's investments in the resources that have a direct impact on these components ( $\mathrm{Y}$ axes in the figure: average investments, coming from the "investment curve" described above, and weighted in accordance with the estimates presented in Exhibit $3)$.

On the right side of the two compasses we can also identify the overall performance levels of the two companies, measured with a "perceived competitive profile in terms of Value" ("pcpV") indicator, which is just the average of the individual performances on the components of value, weighted depending on the importance of these components for the market (see Appendix). The existence of competitors for the calculation of this "pcpV" is totally irrelevant: if, however, competitors do exist, their performance can be estimated exactly in the same way, as suggested by Exhibit 4.

The dotted lines in the figure identify a sort of "ideal" investments, had the companies allocated the sum of the indices representing their actual investments exactly in proportion of the estimated importance assigned by the market to the components of value: in the cases represented in the figure, we can see that company B, compared to company $\mathrm{C}$, invested in a less coherent and selective way, and this generated an actual overall value index ("pcpVa") slightly lower than the "ideal" one ("pcpVi"), given its investment decisions.

However, we can also see that the investment levels of company B are higher than those of company C, and this produces a better "pcpV", probably at the expense of some inefficiencies from a strictly economic standpoint: as a matter of fact, we should also consider a number of other fac- 
tors (briefly summarized below), which, however, are not essential for the point we want to make.

In any case, it seems to us that presenting the companies' strategies with this "compasses", is more exhaustive and meaningful than adopting the "value curves", since the "compass" identifies and distinguishes both the relative importance of the components of value for the market, and the investment levels in the resources which manage these components. Simply stated, it is a way of keeping under control, in conjunction with the other components of the "Strateco Dashboard", the presumably "right" course of action, in any possible ocean, sea, lake, or pond, and in waters of any possible colors (blue, light-blue, purple, reddish, red, etc.).

Always based on these analyses, we can also estimate, for each competitor and each component of value, the difference between "actual" and "ideal" investments: in Exhibit 5 we can see that companies A and B were relatively coherent in terms of "brand", but much less consistent in terms of "service" (too little investment) and "quality" (too much investment), while company C did the best overall job, although with a lower total investment, as we saw in Exhibit 4 above.

\section{EXHIBIT 5}

It is obvious that the algebraic sum of the discrepancies above and below zero is zero, i.e. that the excess resources invested in one or more components of value (always measured with indices) subtract exactly the same resources to other components, which should have deserved more attention: this "opportunity cost" concept is often ignored in the management practice, and, as far as we know, is never considered (and explicitly measured) in any management control system.

3. The value/price ratio and the demand curve. Starting from the value indices described above and an estimated range of variability of prices, we can therefore estimate the value/price ratios (which, in practice, represent indices of competitiveness) and the related demand curves, obviously depending on estimates about its relative elasticity to price vs. value (i.e. in some market contexts, the demand could be relatively more sensitive to price than to value, and vice versa in other contexts). In the presence of competitors, the related value/price ratios could provide a rough estimate of their likely market share.

4. The impact on the creation of economic value. Finally, based on the above estimates, we can measure the impact of the strategy on the creation of value. With appropriate metrics - for example, Rappaport's (1986/1999) shareholder value added (SVA) or other measures (Madden, 1997; Boston Consulting Group, 2008/2001; Weissenrieder, 1998; Damodaran, 2000; Fabozzi \& Grant, 2000; Das et al., 2007; Venanzi, 2010) - we can translate the estimates above into the main value drivers, and therefore measure the value created by the strategy:

- The value/price ratio and the demand curve determine the sales growth rate.

- The investment curve determines the rate of operating profit margin and the rate of incremental capital investment, in terms of rate of (fixed and working) capital intensity of sales.

- The "relative" pcpV/price ratio (i.e. the firm's ability to preserve it from potentially competing emulators) affects the value growth duration, that is the period of competitive advantage during which the firm is expected to earn returns in excess of its cost of capital, and therefore to create value (see the Appendix for the estimate of the operating cash flow).

- All the components of the model and the parameters that define the business contribute to the determination of the cost of capital, i.e. the discount rate of the cash flows and of the terminal value generated by the strategy.

It is therefore easy to assess, with simple sensitivity analyses, which could be the economic results of alternative strategic assumptions, and, hence, their attractiveness and feasibility. By using a metric like the SVA, that better than others focuses on the standard drivers of value (sales growth, operating margins, investments, cost of capital), operating managers can find strategies with the highest potential for increasing value. Thanks to the analytical tools made available by the electronic spreadsheet, we can then identify investment strategies that optimize, alternatively, the $p c p V$, the value/price ratio (hence, where applicable, the market share), and the SVA, other things equal. 
5. The financial feasibility of the relative value/price ratio. Furthermore, the above model allows an evaluation of the strategy's financial feasibility. Based on an estimate of the incremental financial needs (see Appendix), we can compare these to the firm's ability to internally financing them by using the re-investment of the free cash flow, and therefore determine the incremental external capital which could be provided (as debt or equity) in order to implement the strategy (Donaldson, 1985).

We will not go further in-depth on these last three points, since they are straightforward, based on classical economic approaches: we just want to point out that the logical framework described above can be consistent with the well known financial approach of measuring the impact of strategy on value creation. This quantitative approach could be complemented, especially in the case of new market spaces, by some qualitative approaches suggested in the BOS text.

Furthermore, we will not consider here other important aspects in the design of a strategy, such as the impact of what we call "indirect tools" (information, control, and reward systems, etc.), nor we will consider the impact of professional profiles on the effectiveness and efficiency of the investments in both the direct and indirect tools, since this goes beyond the purpose of this article, mainly aimed at suggesting a more feasible and practical approach to strategy development for SMEs.

\section{Testing The New Approach}

We already adopted this new approach, complemented with the just mentioned additional diagnostic tools, for analyzing and designing competitive strategies in several different industry contexts. However, testing its ability to "predict" winning strategies would require appropriate experimental conditions and controls, that are difficult and costly to design and implement in a relatively reasonable time frame.

We therefore decided to experiment the approach in a "laboratory" setting, developing a strategic management simulation that included some additional features (impact of "indirect tools" and professional profiles, dynamic and carryover effects over time of the previous years' market positions), and testing our diagnostic models with different groups of managers, in relation to two different and hypothetical B2B industry scenarios ("mass" and "customized" industrial products).

In particular, we recently conducted a two-day management workshop in a medium-size Italian firm (www.landi.it), which manufactures and sells worldwide, to major auto OEMs, LPG and methane engine driven systems. Based on an explicit description, although just qualitative for the participants, of the market and competitive characteristics of the two "virtual" businesses, we found that the simulated company that obtained the best results, in terms of both profitability and market share, at the end of a four-year simulated period, was represented by the management team that, within each business, had consistently minimized the discrepancies between actual and ideal investments, while maintaining the highest value/price ratios in both of them (these performances are not automatically linked together in the underlying model).

This result could obviously be explained by the intrinsic logic of the model (although not "visible" by the participants). However, considering:

- the broadly positive reactions of the participating managers in terms of better awareness of the relationships among the relevant variables

- the possibility of adjusting all the major parameters of the simulation, depending on the judgment and industry experience of the interested users,

we think that, in the worst case, the Strateco Dashboard could represent a powerful diagnostic tool for assessing the likely attractiveness of alternative strategies, based on explicit assumptions about the market behavior, made by the decision makers.

\section{Potential Weaknesses of the "Dashboard" and its Components}

As we have seen above, for the sake of simplicity, in the description of the conceptual model behind our framework we resorted to some shortcuts. In particular: 
- We assumed a unique configuration (vector) of the tools' relative importance (weights) for each choice criterion: however, alternative mixes of resources could possibly produce similar combinations of perceived value for the market and profitability for the firm.

- We assumed a linear relationship between investment and results, within the min-max range of investment considered "reasonable" in the industry of interest, and this could not be the case, especially in highly competitive market contexts.

- We assumed that the tools used by firms for managing these criteria are independent from each other, and this could obviously be false in a real-life situation: for example, the combined investments in R\&D and raw materials could produce a synergistic effect that is larger than the sum of the individual impacts of the two investments.

These limitations can be overcome with a more sophisticated approach, that takes into consideration alternative mixes of resources, non-linear relationships between investments and results, and the potential interactions within tools (see an example of a possible solution in the Appendix). Obviously, the assumption of interaction could also be extended to the choice criteria (here considered independent from each other), but we would incur the risk of making the model unmanageable in terms of provision of inputs. This enhanced model could be more suitable for customizing the dashboard depending on the market and competitive characteristics of specific industry contexts. However, the logic of the underlying conceptual approach would not change.

\section{CONCLUSION: THE SPECIFIC RELEVANCE OF THE SUGGESTED APPROACH FOR SMES}

Looking at the above model, it is easy to see how its characteristics and guidelines can support most of the strategic requirements listed at the end of the introductory part of this article. In particular, the Strateco Dashboard allows and requires the following methodological approaches:

- explicit identification of the components of value that are more relevant for the market, and assessment of their relative importance: this could prevent from pursuing, for example, product characteristics that could not anesthetize the demand's elasticity to price

- $\quad$ explicit identification, especially critical for SMEs, of the needed and available resources for satisfying the market's requirements, and assessment of their relative importance for managing the most relevant components of value

- $\quad$ explicit assessment of alternative strategic approaches for delivering promising value/price ratios ..... and, even more important for SMEs:

- possibility of optimizing the allocation of the available resources, based on reasonable assumptions and estimates about their relative effectiveness, also in consideration of market and competitive constraints

- $\quad$ possibility, which is normally precluded by most management control systems (or all of them?), of explicitly assessing the coherence between the selected options and the formulated assumptions. 


\section{APPENDIX}

\section{Exhibit 1}

\section{STRATEGIES OF TWO MAJOR "STRATEGIC GROUPS" VS. THAT OF [YELLOW TAIL]}

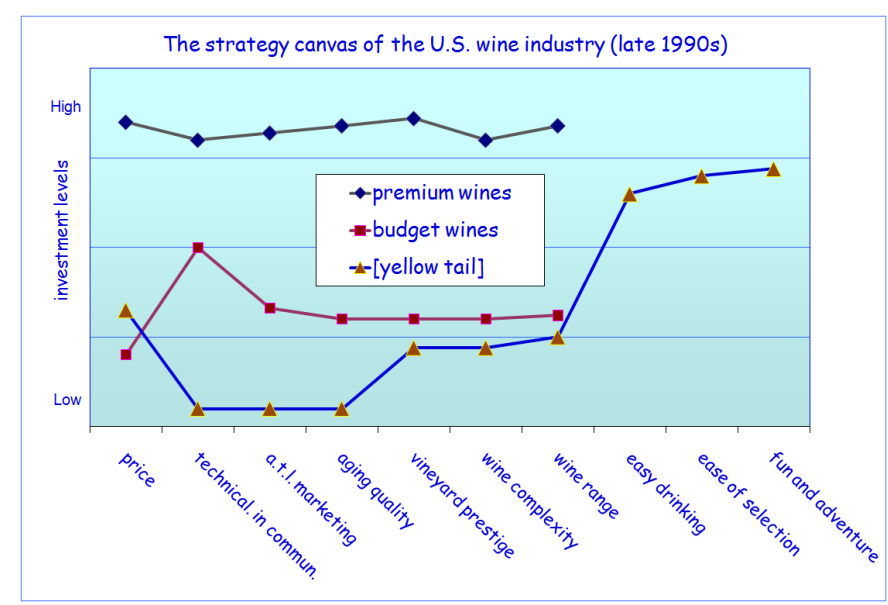

(adapted from Kim \& Mauborgne)

\section{Exhibit 2}

In the example depicted in the figure, company B "invested", respectively in raw materials, $\mathbf{R} \& \mathbf{D}$, and production, $€ 0.30$ per kilo (within an estimated min-max range of $€ 0.20-0.40$ ), $€ 100 \mathrm{k}$ (range $40 \mathrm{k}-180 \mathrm{k}$ ), and $€ 175 \mathrm{k}$ (range $80 \mathrm{k}-260 \mathrm{k}$ ), obtaining, on a scale from 1 to 10 , indices of 5.5, 4.9, and 5.8 respectively.

\section{Exhibit 4}

Always referring to company $B$ and its investment levels of 5.5, 4.9, and 5.8 in raw materials, R\&D, and production, and assuming a relative impact on quality of these resources of 40,30 , and $30 \%$ respectively (Exhibit 3 ), the overall performance on quality of company B is measurable with an index of 5.4.

The same reasoning applies to the other components of value: in practice, company B's actual performance in terms of value (thanks to the various investments or expenses) is respectively equal to 5.4, 6.0 and 7.1 on quality, image, and service, which generates an overall "actual" value index ("pcpVa") of 6.4 (weighted, depending on the relative importance of these components for the market: respectively, 20,30, and 50\%).

\section{Estimate of the operating cash flow}

The operating cash flow at a generic period $t$ can be estimated as follows: Sales ${ }_{t-1} *(1+$ growth rate of sales) * rate of operating profit margin * $\left(1-\right.$ tax rate $^{-}-\left(\right.$sales $_{t}$ - sales $\left.{ }_{t-1}\right) *$ rate of incremental investment in fixed assets and working capital. At the end of the projected period the terminal value can be determined as a perpetuity of the net operating cash flow at the horizon, assuming a steadystate beyond this term. Cash flows and terminal value are therefore discounted by the cost of capital.

\section{Estimate of the incremental financial needs}

We can estimate the incremental financial needs as follows:

Sales $t_{t}$ growth rate of sales $*$ rate of incremental investment in fixed assets and working capital.

\section{Considering the interactions among tools}

Let us consider two different tools, such as R\&D and raw materials, and assume that the effectiveness of an investment in the latter depends on different levels (or ranges) of investment in the former: we could therefore estimate as many different S-shaped curves for the relationship between raw materials and results in terms of contribution to quality, as ranges of investments in R\&D. 


\section{REFERENCES}

Abell, D.F., (1980), Defining the business. The starting point of strategic planning, Prentice Hall. Inc., Englewood Cliffs.

Beckwith, H., (1997), Selling the invisible, Warner Books.

Boston Consulting Group, (2008), Missing link - Focusing corporate strategy on value creation, The 2008 Value Creators Report.

(2001), Dealing with investors' expectations - A global study of company valuations and their strategic implications, The 2001 Value Creators Report.

Coltorti, F., (2010), Italian medium-sized industrial companies, Mediobanca-Unioncamere Conference on Medium-size Italian Businesses, Milan, March 11, 2010.

, (2009), Medium-sized firms, groups and industrial districts: An Italian perspective, in Becattini G., Bellandi M., and De Propris L. (eds.), A Handbook of Industrial Districts, Edward Elgar.

----------, (2008), Prolusione Master per Imprenditori di Piccole e Medie imprese, Fondazione CUOA, I Quaderni, n.4.

Confindustria, Ricerche e Studi - R\&S and Unioncamere, (2010), Medium-sized Enterprises in Europe.

Damodaran, A., (2000), Value Creation and Enhancement: Back to the Future, Stern School of Business.

Das, D., S. Thomas, and R. Repetto, (2007), Integrated Environmental and Financial Performance Metrics for Investment Analysis and Portfolio Management, Corporate Governance: An International Review, 15(3).

Donaldson, G., (1985), Financial goals and strategic consequences, Harvard Business Review, 28.

Fabozzi, F. J., and J. L. Grant (eds.), (2000), Value-Based Metrics: Foundations and Practice, John Wiley \& Sons.

Gagliardi, C., (2010), Le medie imprese-Il Made in Italy che guida la ripresa, MediobancaUnioncamere Conference on Medium-size Italian Businesses, Milan, March 11.

Gandellini, G., (2010), Mass \& Custom: a strategic management simulation, University of Roma Tre.

Gandellini, G, and Venanzi, D., (2011), Purple Ocean Strategy, ... i.e. Blue Ocean Revisited, Working Paper, University of Roma Tre.

Harrington, H.J., (1987), The improvement process, McGraw-Hill.

Howard, J. and Sheth, J., (1969), Theory of buyer behavior, John Wiley \& Sons.

Kaplan, R.S. and Norton, D.P., (1996), The Balanced Scorecard, Harvard Business School Press.

Kim, W. C. and R. Mauborgne, (2005), Blue Ocean Strategy: How to Create Uncontested Market Space and Make the Competition Irrelevant, Harvard Business School Press.

Levitt, T., (1975), Marketing Myopia, Harvard Business Review, 5.

Madden, B.J., (2007), For better corporate governance. The shareholder value review, Journal of Applied Corporate Finance, 19 (1).

Porter, M., (1996), What is Strategy?, Harvard Business Review, November-December.

Rappaport, A., (1986), Creating Shareholder Value: A guide for managers and investors, Free Press. Review, 2 . (1999), New thinking on how to link executive pay with performance, Harvard Business

Venanzi, D., 2010, Financial performance measures and value creation: a review, SSRN WP, http:// ssrn.com/abstract $=1716209$.

Weissenrieder, F., (1998), Value based management: Economic value added or cash value added?, Gothenburg studies in Financial Economics, 971214. 